\title{
The analytic continuation of the high-energy quark-quark scattering amplitude
}

\author{
Enrico Meggiolaro \\ Dipartimento di Fisica, \\ Università di Pisa, \\ and INFN, Sezione di Pisa, \\ I-56100 Pisa, Italy.
}

\begin{abstract}
It is known that the high-energy quark-quark scattering amplitude can be described by the expectation value of two lightlike Wilson lines, running along the classical trajectories of the two colliding particles. Generalizing the results of a previous paper, we give here the general proof that the expectation value of two infinite Wilson lines, forming a certain hyperbolic angle in Minkowski space-time, and the expectation value of two infinite Euclidean Wilson lines, forming a certain angle in Euclidean four-space, are connected by an analytic continuation in the angular variables. This result could be used to evaluate the high-energy scattering amplitude directly on the lattice.
\end{abstract}




\section{Introduction}

It is well known that the quark-quark scattering amplitude, at high squared energies $s$ in the center of mass and small squared transferred momentum $t$ (that is $s \rightarrow \infty$ and $|t| \ll s$, let us say $|t| \leq 1 \mathrm{GeV}^{2}$ ), can be described by the expectation value of two lightlike Wilson lines, running along the classical trajectories of the two colliding particles [1] [2].

In the center-of-mass reference system (c.m.s.), taking for example the initial trajectories of the two quarks along the $x^{1}$-axis, the scattering amplitude has the following form [explicitly indicating the color indices $(i, j, \ldots)$ and the spin indices $(\alpha, \beta, \ldots)$ of the quarks]

$$
\begin{aligned}
M_{f i} & =\left\langle\psi_{i \alpha}\left(p_{1}^{\prime}\right) \psi_{k \gamma}\left(p_{2}^{\prime}\right)|M| \psi_{j \beta}\left(p_{1}\right) \psi_{l \delta}\left(p_{2}\right)\right\rangle \\
& \underset{s \rightarrow \infty}{\sim}-\frac{i}{Z_{\psi}^{2}} \cdot \delta_{\alpha \beta} \delta_{\gamma \delta} \cdot 2 s \int d^{2} \mathbf{z}_{t} e^{i \mathbf{q} \cdot \mathbf{z}_{t}}\left\langle\left[W_{1}\left(z_{t}\right)-\mathbf{1}\right]_{i j}\left[W_{2}(0)-\mathbf{1}\right]_{k l}\right\rangle,
\end{aligned}
$$

where $q=(0,0, \mathbf{q})$, with $t=q^{2}=-\mathbf{q}^{2}$, is the tranferred four-momentum and $z_{t}=$ $\left(0,0, \mathbf{z}_{t}\right)$, with $\mathbf{z}_{t}=\left(z^{2}, z^{3}\right)$, is the distance between the two trajectories in the transverse plane [the coordinates $\left(x^{0}, x^{1}\right)$ are often called longitudinal coordinates]. The expectation value $\langle f(A)\rangle$ is the average of $f(A)$ in the sense of the functional integration over the gluon field $A^{\mu}$ (including also the determinant of the fermion matrix, i.e., $\operatorname{det}\left[i \gamma^{\mu} D_{\mu}-m\right]$, where $D^{\mu}=\partial^{\mu}+i g A^{\mu}$ is the covariant derivative) [1] [2]. The two lightlike Wilson lines $W_{1}\left(z_{t}\right)$ and $W_{2}(0)$ in Eq. (1.1) are defined as

$$
\begin{aligned}
W_{1}\left(z_{t}\right) & =P \exp \left[-i g \int_{-\infty}^{+\infty} A_{\mu}\left(z_{t}+p_{1} \tau\right) p_{1}^{\mu} d \tau\right] ; \\
W_{2}(0) & =P \exp \left[-i g \int_{-\infty}^{+\infty} A_{\mu}\left(p_{2} \tau\right) p_{2}^{\mu} d \tau\right],
\end{aligned}
$$

where $P$ stands for "path ordering" and $A_{\mu}=A_{\mu}^{a} T^{a} ; p_{1} \simeq\left(E, E, \mathbf{0}_{t}\right)$ and $p_{2} \simeq\left(E,-E, \mathbf{0}_{t}\right)$ are the initial four-momenta of the two quarks. The space-time configuration of these two Wilson lines is shown in Fig. 1.

Finally, $Z_{\psi}$ in Eq. (1.1) is the fermion-field renormalization constant, which can be written in the eikonal approximation as [1]

$$
Z_{\psi} \simeq \frac{1}{N_{c}}\left\langle\operatorname{Tr}\left[W_{1}\left(z_{t}\right)\right]\right\rangle=\frac{1}{N_{c}}\left\langle\operatorname{Tr}\left[W_{1}(0)\right]\right\rangle=\frac{1}{N_{c}}\left\langle\operatorname{Tr}\left[W_{2}(0)\right]\right\rangle
$$




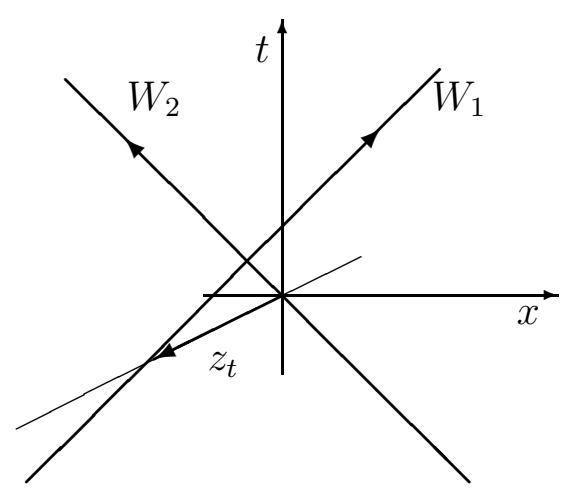

Fig. 1. The space-time configuration of the two lightlike Wilson lines $W_{1}$ and $W_{2}$ entering in the expression (1.1) for the high-energy quark-quark elastic scattering amplitude.

where $N_{c}$ is the number of colours.

In what follows, we shall deal with the quantity

$$
g_{M(i j, k l)}(s ; t) \equiv \frac{1}{Z_{\psi}^{2}} \int d^{2} \mathbf{z}_{t} e^{i \mathbf{q} \cdot \mathbf{z}_{t}}\left\langle\left[W_{1}\left(z_{t}\right)-\mathbf{1}\right]_{i j}\left[W_{2}(0)-\mathbf{1}\right]_{k l}\right\rangle
$$

in terms of which the scattering amplitude can be written as

$$
M_{f i}=\left\langle\psi_{i \alpha}\left(p_{1}^{\prime}\right) \psi_{k \gamma}\left(p_{2}^{\prime}\right)|M| \psi_{j \beta}\left(p_{1}\right) \psi_{l \delta}\left(p_{2}\right)\right\rangle \underset{s \rightarrow \infty}{\sim}-i \cdot 2 s \cdot \delta_{\alpha \beta} \delta_{\gamma \delta} \cdot g_{M(i j, k l)}(s ; t)
$$

The quantity $g_{M(i j, k l)}(s ; t)$ depends not only on $t=-\mathbf{q}^{2}$, but also on $s$. In fact, as was pointed out by Verlinde and Verlinde in [3], it is a singular limit to take the Wilson lines in (1.4) exactly lightlike. A way to regularize this sort of "infrared" divergence (so called because it essentially comes from the limit $m \rightarrow 0$, where $m$ is the quark mass) consists in letting each line have a small timelike component, so that they coincide with the classical trajectories for quarks with a finite mass $m$ (see also Ref. [幽 for a discussion about this point). In other words, one first evaluates the quantity $g_{M(i j, k l)}(\beta ; t)$ for two Wilson lines along the trajectories of two quarks (with mass $m$ ) moving with velocity $\beta$ and $-\beta$ $(0<\beta<1)$ in the $x^{1}$-direction. This is equivalent to consider two infinite Wilson lines forming a certain (finite) hyperbolic angle $\chi$ in Minkowski space-time. Then, to obtain the correct high-energy scattering amplitude, one has to perform the limit $\beta \rightarrow 1$, that 
is $\chi \rightarrow \infty$, in the expression for $g_{M(i j, k l)}(\beta ; t)$ :

$$
M_{f i}=\left\langle\psi_{i \alpha}\left(p_{1}^{\prime}\right) \psi_{k \gamma}\left(p_{2}^{\prime}\right)|M| \psi_{j \beta}\left(p_{1}\right) \psi_{l \delta}\left(p_{2}\right)\right\rangle \underset{s \rightarrow \infty}{\sim}-i \cdot 2 s \cdot \delta_{\alpha \beta} \delta_{\gamma \delta} \cdot g_{M(i j, k l)}(\beta \rightarrow 1 ; t) .
$$

Proceeding in this way one obtains a $\ln s$ dependence of the amplitude, as expected from ordinary perturbation theory and as confirmed by the experiments on hadron-hadron scattering processes [5] [6]. In Sect. 3 of Ref. [4] we have followed this procedure to explicitly evaluate the second member of (1.6) up to the fourth order in the expansion in the renormalized coupling constant: the results so derived are in agreement with those obtained from ordinary perturbation theory.

The direct evaluation of the expectation value (1.4) is a highly non-trivial matter and it is strictly connected with the ultraviolet properties of Wilson-line operators [7] [8]. Some non-perturbative approaches for the calculation of (1.4) have been proposed in Refs. [9] and [10].

In a recent paper [4] we have proposed a new approach, which consists in adapting the scattering amplitude to the Euclidean world: this approach could open the way for the direct evaluation of the scattering amplitude on the lattice. More explicitly, in Ref. [4] we have given arguments showing that the expectation value of two infinite Wilson lines, forming a certain hyperbolic angle in Minkowski space-time, and the expectation value of two infinite Euclidean Wilson lines, forming a certain angle in Euclidean four-space, are likely to be connected by an analytic continuation in the angular variables. This relation of analytic continuation has been proven in Ref. [4] for an Abelian gauge theory (QED) in the so-called quenched approximation and for a non-Abelian gauge theory (QCD) up to the fourth order in the renormalized coupling constant in perturbation theory: a general proof was missing up to now.

In this paper, we shall generalize the results of Ref. [4] and give the rigorous proof of the above-mentioned relation of analytic continuation for a non-Abelian gauge theory with gauge group $S U\left(N_{c}\right)$ [as well as for an Abelian gauge theory (QED)]. The approach adopted in Ref. [4] consisted in explicitly evaluating the amplitudes $g_{M}(\chi ; t)$ and $g_{E}(\theta ; t)$, in the Minkowski and the Euclidean world, in some given approximation (such as the quenched approximation) or up to some order in perturbation theory and in finally comparing the two expressions so obtained. Instead, in this paper we shall give a general proof, which essentially exploits the relation between the gluonic Green functions in the two theories. 


\section{From Minkowskian to Euclidean theory}

Let us consider the following quantity, defined in Minkowski space-time:

$$
\begin{aligned}
g_{M}\left(p_{1}, p_{2} ; t\right) & =\frac{M\left(p_{1}, p_{2} ; t\right)}{Z_{W}^{2}}, \\
M\left(p_{1}, p_{2} ; t\right) & =\int d^{2} \mathbf{z}_{t} e^{i \mathbf{q} \cdot \mathbf{z}_{t}}\left\langle\left[W_{1}\left(z_{t}\right)-\mathbf{1}\right]_{i j}\left[W_{2}(0)-\mathbf{1}\right]_{k l}\right\rangle,
\end{aligned}
$$

where $p_{1}$ and $p_{2}$ are the four-momenta [lying (for example) in the plane $\left(x^{0}, x^{1}\right)$ ], which define the trajectories of the two Wilson lines $W_{1}$ and $W_{2}\left(A_{\mu}=A_{\mu}^{a} T^{a}\right.$ and $m$ is the fermion mass):

$$
\begin{aligned}
W_{1}\left(z_{t}\right) & \equiv P \exp \left[-i g \int_{-\infty}^{+\infty} A_{\mu}\left(z_{t}+\frac{p_{1}}{m} \tau\right) \frac{p_{1}^{\mu}}{m} d \tau\right] ; \\
W_{2}(0) & \equiv P \exp \left[-i g \int_{-\infty}^{+\infty} A_{\mu}\left(\frac{p_{2}}{m} \tau\right) \frac{p_{2}^{\mu}}{m} d \tau\right] .
\end{aligned}
$$

$Z_{W}$ in Eq. (2.1) is defined as ( $N_{c}$ being the number of colours)

$$
Z_{W} \equiv \frac{1}{N_{c}}\left\langle\operatorname{Tr}\left[W_{1}\left(z_{t}\right)\right]\right\rangle=\frac{1}{N_{c}}\left\langle\operatorname{Tr}\left[W_{1}(0)\right]\right\rangle=\frac{1}{N_{c}}\left\langle\operatorname{Tr}\left[W_{2}(0)\right]\right\rangle
$$

(The two last equalities come from the Poincaré invariance.) This is a sort of Wilsonline's renormalization constant: as shown in Ref. [1], $Z_{W}$ coincides with the fermion renormalization constant $Z_{\psi}$ in the eikonal approximation.

By virtue of the Lorentz symmetry, we can define $p_{1}$ and $p_{2}$ in the c.m.s. of the two particles, moving with speed $\beta$ and $-\beta$ along the $x^{1}$-direction:

$$
\begin{aligned}
p_{1}^{\mu} & =E\left(1, \beta, \mathbf{0}_{t}\right), \\
p_{2}^{\mu} & =E\left(1,-\beta, \mathbf{0}_{t}\right),
\end{aligned}
$$

where $E=m / \sqrt{1-\beta^{2}}$ (in units with $c=1$ ) is the energy of each particle (so that: $\left.s=4 E^{2}\right)$.

We now introduce the hyperbolic angle $\psi$ [in the plane $\left(x^{0}, x^{1}\right)$ ] of the trajectory of $W_{1}$ : it is given by $\beta=\tanh \psi$. We can give the explicit form of the Minkowski four-vectors $u_{1}=p_{1} / m$ and $u_{2}=p_{2} / m$ in terms of the hyperbolic angle $\psi$ :

$$
\begin{aligned}
& u_{1}=\frac{p_{1}}{m}=\left(\cosh \psi, \sinh \psi, \mathbf{0}_{t}\right), \\
& u_{2}=\frac{p_{2}}{m}=\left(\cosh \psi,-\sinh \psi, \mathbf{0}_{t}\right) .
\end{aligned}
$$


Clearly, $u_{1}^{2}=u_{2}^{2}=1$ and

$$
u_{1} \cdot u_{2}=\cosh (2 \psi)=\cosh \chi,
$$

where $\chi=2 \psi$ is the hyperbolic angle [in the plane $\left(x^{0}, x^{1}\right)$ ] between the two trajectories of $W_{1}$ and $W_{2}$.

In an analogous way, we can consider the following quantity, defined in Euclidean space-time:

$$
\begin{aligned}
g_{E}\left(p_{1 E}, p_{2 E} ; t\right) & =\frac{E\left(p_{1 E}, p_{2 E} ; t\right)}{Z_{W E}^{2}}, \\
E\left(p_{1 E}, p_{2 E} ; t\right) & =\int d^{2} \mathbf{z}_{t} e^{i \mathbf{q} \cdot \mathbf{z}_{t}}\left\langle\left[W_{1 E}\left(z_{t E}\right)-\mathbf{1}\right]_{i j}\left[W_{2 E}(0)-\mathbf{1}\right]_{k l}\right\rangle_{E},
\end{aligned}
$$

where $z_{t E}=\left(z_{1}, z_{2}, z_{3}, z_{4}\right)=\left(0, \mathbf{z}_{t}, 0\right)$ and $q_{E}=(0, \mathbf{q}, 0)$ (so that: $q_{E}^{2}=\mathbf{q}^{2}=-t$ ). The expectation value $\langle\ldots\rangle_{E}$ must be intended now as a functional integration with respect to the gauge variable $A_{\mu}^{(E)}=A_{\mu}^{(E) a} T^{a}$ in the Euclidean theory. The Euclidean four-vectors $p_{1 E}$ and $p_{2 E}$ [lying (for example) in the plane $\left.\left(x_{1}, x_{4}\right)\right]$ define the trajectories of the two Euclidean Wilson lines $W_{1 E}$ and $W_{2 E}$ :

$$
\begin{aligned}
W_{1 E}\left(z_{t E}\right) & \equiv P \exp \left[-i g \int_{-\infty}^{+\infty} A_{\mu}^{(E)}\left(z_{t E}+p_{1 E} \tau\right) p_{1 E \mu} d \tau\right] \\
W_{2 E}(0) & \equiv P \exp \left[-i g \int_{-\infty}^{+\infty} A_{\mu}^{(E)}\left(p_{2 E} \tau\right) p_{2 E \mu} d \tau\right] .
\end{aligned}
$$

$Z_{W E}$ in Eq. (2.7) is defined analogously to $Z_{W}$ in Eq. (2.3):

$$
Z_{W E} \equiv \frac{1}{N_{c}}\left\langle\operatorname{Tr}\left[W_{1 E}\left(z_{t E}\right)\right]\right\rangle=\frac{1}{N_{c}}\left\langle\operatorname{Tr}\left[W_{1 E}(0)\right]\right\rangle=\frac{1}{N_{c}}\left\langle\operatorname{Tr}\left[W_{2 E}(0)\right]\right\rangle .
$$

(The two last equalities come from the $O(4)$ plus translation invariance.)

We can now expand the Wilson lines $W_{1}$ and $W_{2}$ in power series of the coupling constant $g$ and take the pieces with $g^{n}$ and $g^{r}$ respectively. Their contribution to the amplitude $M\left(p_{1}, p_{2} ; t\right)$ will be called $M_{(n, r)}\left(p_{1}, p_{2} ; t\right)$ (so that $\left.M=\sum_{n=1}^{\infty} \sum_{r=1}^{\infty} M_{(n, r)}\right)$ and is given by

$$
\begin{aligned}
& M_{(n, r)}\left(p_{1}, p_{2} ; t\right)=(-i g)^{(n+r)}\left(T^{a_{1}} \ldots T^{a_{n}}\right)_{i j}\left(T^{b_{1}} \ldots T^{b_{r}}\right)_{k l} \int d^{2} \mathbf{z}_{t} e^{i \mathbf{q} \cdot \mathbf{z}_{t}} \times \\
& \int d \tau_{1} \frac{p_{1}^{\mu_{1}}}{m} \ldots \int d \tau_{n} \frac{p_{1}^{\mu_{n}}}{m} \int d \omega_{1} \frac{p_{2}^{\nu_{1}}}{m} \ldots \int d \omega_{r} \frac{p_{2}^{\nu_{r}}}{m} \times \\
& \theta\left(\tau_{n}-\tau_{n-1}\right) \ldots \theta\left(\tau_{2}-\tau_{1}\right) \theta\left(\omega_{r}-\omega_{r-1}\right) \ldots \theta\left(\omega_{2}-\omega_{1}\right) \times \\
& \left\langle A_{\mu_{1}}^{a_{1}}\left(z_{t}+\frac{p_{1}}{m} \tau_{1}\right) \ldots A_{\mu_{n}}^{a_{n}}\left(z_{t}+\frac{p_{1}}{m} \tau_{n}\right) A_{\nu_{1}}^{b_{1}}\left(\frac{p_{2}}{m} \omega_{1}\right) \ldots A_{\nu_{r}}^{b_{r}}\left(\frac{p_{2}}{m} \omega_{r}\right)\right\rangle .
\end{aligned}
$$


The corresponding quantity for the Euclidean theory $E_{(n, r)}\left(p_{1 E}, p_{2 E} ; t\right)$, obtained taking the pieces with $g^{n}$ and $g^{r}$ in the expansion of the Euclidean Wilson lines $W_{1 E}$ and $W_{2 E}$ inside $E\left(p_{1 E}, p_{2 E} ; t\right)$, is given by

$$
\begin{aligned}
& E_{(n, r)}\left(p_{1 E}, p_{2 E} ; t\right)=(-i g)^{(n+r)}\left(T^{a_{1}} \ldots T^{a_{n}}\right)_{i j}\left(T^{b_{1}} \ldots T^{b_{r}}\right)_{k l} \int d^{2} \mathbf{z}_{t} e^{i \mathbf{q} \cdot \mathbf{z}_{t}} \times \\
& \quad \int d \tau_{1} p_{1 E}^{\mu_{1}} \ldots \int d \tau_{n} p_{1 E}^{\mu_{n}} \int d \omega_{1} p_{2 E}^{\nu_{1}} \ldots \int d \omega_{r} p_{2 E}^{\nu_{r}} \theta\left(\tau_{n}-\tau_{n-1}\right) \ldots \theta\left(\tau_{2}-\tau_{1}\right) \times \\
& \quad \theta\left(\omega_{r}-\omega_{r-1}\right) \ldots \theta\left(\omega_{2}-\omega_{1}\right)\left\langle A_{(E) \mu_{1}}^{a_{1}}\left(z_{t E}+p_{1 E} \tau_{1}\right) \ldots A_{(E) \mu_{n}}^{a_{n}}\left(z_{t E}+p_{1 E} \tau_{n}\right) \times\right. \\
& \left.\quad \times A_{(E) \nu_{1}}^{b_{1}}\left(p_{2 E} \omega_{1}\right) \ldots A_{(E) \nu_{r}}^{b_{r}}\left(p_{2 E} \omega_{r}\right)\right\rangle_{E} .
\end{aligned}
$$

It is known that, making use of the correspondence

$$
\begin{array}{lll}
A_{0}(x) \rightarrow i A_{4}^{(E)}\left(x_{E}\right) & , \quad & A_{k}(x) \rightarrow A_{k}^{(E)}\left(x_{E}\right) \\
\text { with }: & x^{0} \rightarrow-i x_{E 4} \quad, & \mathbf{x} \rightarrow \mathbf{x}_{E},
\end{array}
$$

between the Minkowski and the Euclidean world, the following relationship is derived between the gluonic Green functions in the two theories:

$$
\begin{aligned}
& \tilde{B}_{(1)}^{\mu_{1}} \ldots \tilde{B}_{(N)}^{\mu_{N}}\left\langle A_{\mu_{1}}^{a_{1}}\left(\tilde{x}_{(1)}\right) \ldots A_{\mu_{N}}^{a_{N}}\left(\tilde{x}_{(N)}\right)\right\rangle= \\
& \quad=B_{(1) E \mu_{1}} \ldots B_{(N) E \mu_{N}}\left\langle A_{(E) \mu_{1}}^{a_{1}}\left(x_{(1) E}\right) \ldots A_{(E) \mu_{N}}^{a_{N}}\left(x_{(N) E}\right)\right\rangle_{E}
\end{aligned}
$$

where $x_{(i) E}=\left(\mathbf{x}_{(i) E}, x_{(i) E 4}\right)$ are Euclidean four-coordinates and $B_{(i) E}=\left(\mathbf{B}_{(i) E}, B_{(i) E 4}\right)$ are any Euclidean four-vectors, while $\tilde{x}_{(i)}$ and $\tilde{B}_{(i)}$ are Minkowski four-vectors defined as

$$
\begin{gathered}
\tilde{x}_{(i)}=\left(\tilde{x}_{(i)}^{0}, \tilde{\mathbf{x}}_{(i)}\right)=\left(-i x_{(i) E 4}, \mathbf{x}_{(i) E}\right), \\
\tilde{B}_{(i)}=\left(\tilde{B}_{(i)}^{0}, \tilde{\mathbf{B}}_{(i)}\right)=\left(-i B_{(i) E 4}, \mathbf{B}_{(i) E}\right) .
\end{gathered}
$$

For example, in the case $N=2$, if one defines the gluonic propagators as

$$
\begin{aligned}
G_{\mu \nu}^{a b}(x, y) & \equiv\left\langle A_{\mu}^{a}(x) A_{\nu}^{b}(y)\right\rangle, \\
G_{(E) \mu \nu}^{a b}\left(x_{E}, y_{E}\right) & \equiv\left\langle A_{(E) \mu}^{a}\left(x_{E}\right) A_{(E) \nu}^{b}\left(y_{E}\right)\right\rangle_{E},
\end{aligned}
$$

one finds that

$$
\begin{aligned}
G_{00}^{a b}(\tilde{x}, \tilde{y}) & =-G_{(E) 44}^{a b}\left(x_{E}, y_{E}\right) \\
G_{0 j}^{a b}(\tilde{x}, \tilde{y}) & =i G_{(E) 4 j}^{a b}\left(x_{E}, y_{E}\right) \\
G_{j 0}^{a b}(\tilde{x}, \tilde{y}) & =i G_{(E) j 4}^{a b}\left(x_{E}, y_{E}\right) \\
G_{j k}^{a b}(\tilde{x}, \tilde{y}) & =G_{(E) j k}^{a b}\left(x_{E}, y_{E}\right)
\end{aligned}
$$


where $j, k=1,2,3$ are indices for the spatial components and $\tilde{x}$ and $\tilde{y}$ are defined as in Eq. (2.14). From these relations, one immediately derives Eq. (2.13) for $N=2$, with $\tilde{B}$ defined as in Eq. (2.14). The result can be trivially generalized to every $N$.

In our specific case, we can use Eq. (2.13) to state that

$$
\begin{aligned}
\frac{\tilde{p}_{1}^{\mu_{1}}}{m} & \ldots \frac{\tilde{p}_{1}^{\mu_{n}}}{m} \frac{\tilde{p}_{2}^{\nu_{1}}}{m} \ldots \frac{\tilde{p}_{2}^{\nu_{r}}}{m}\left\langle A_{\mu_{1}}^{a_{1}}\left(z_{t}+\frac{\tilde{p}_{1}}{m} \tau_{1}\right) \ldots A_{\mu_{n}}^{a_{n}}\left(z_{t}+\frac{\tilde{p}_{1}}{m} \tau_{n}\right) A_{\nu_{1}}^{b_{1}}\left(\frac{\tilde{p}_{2}}{m} \omega_{1}\right) \ldots A_{\nu_{r}}^{b_{r}}\left(\frac{\tilde{p}_{2}}{m} \omega_{r}\right)\right\rangle= \\
& =\frac{p_{1 E}^{\mu_{1}}}{m} \ldots \frac{p_{1 E}^{\mu_{n}}}{m} \frac{p_{2 E}^{\nu_{1}}}{m} \ldots \frac{p_{2 E}^{\nu_{r}}}{m}\left\langle A_{(E) \mu_{1}}^{a_{1}}\left(z_{t E}+\frac{p_{1 E}}{m} \tau_{1}\right) \ldots A_{(E) \mu_{n}}^{a_{n}}\left(z_{t E}+\frac{p_{1 E}}{m} \tau_{n}\right) \times\right. \\
& \left.\times A_{(E) \nu_{1}}^{b_{1}}\left(\frac{p_{2 E}}{m} \omega_{1}\right) \ldots A_{(E) \nu_{r}}^{b_{r}}\left(\frac{p_{2 E}}{m} \omega_{r}\right)\right\rangle_{E}
\end{aligned}
$$

where $p_{i E}=\left(\mathbf{p}_{i E}, p_{i E 4}\right)$, for $i=1,2$, are two Euclidean four-vectors and $\tilde{p}_{i}$ are the two corresponding Minkowski four-vectors, obtained according to Eq. (2.14):

$$
\tilde{p}_{i}=\left(\tilde{p}_{i}^{0}, \tilde{\mathbf{p}}_{i}\right)=\left(-i p_{i E 4}, \mathbf{p}_{i E}\right)
$$

By virtue of the definitions (2.10) and (2.11) for $M_{(n, r)}$ and $E_{(n, r)}$ respectively, Eq. (2.17) implies that:

$$
E_{(n, r)}\left(\frac{p_{1 E}}{m}, \frac{p_{2 E}}{m} ; t\right)=M_{(n, r)}\left(\tilde{p}_{1}, \tilde{p}_{2} ; t\right) .
$$

This relation is valid for every couple of integer numbers $(n, r)$, so that, more generally:

$$
E\left(\frac{p_{1 E}}{m}, \frac{p_{2 E}}{m} ; t\right)=M\left(\tilde{p}_{1}, \tilde{p}_{2} ; t\right)
$$

Of course $M$, considered as a general function of $p_{1}, p_{2}$ [and $\left.q=(0,0, \mathbf{q})\right]$, can only depend on the scalar quantities constructed with the vectors $p_{1}, p_{2}$ and $q=(0,0, \mathbf{q})$ : the only possibilities are $q^{2}=-\mathbf{q}^{2}=t, p_{1} \cdot p_{2}, p_{1}^{2}$ and $p_{2}^{2}$, since $p_{1} \cdot q=p_{2} \cdot q=0$. Moreover, it is clear from the definitions (2.1) and (2.2) that $M$ cannot depend on the (positive) normalizations of the four-vectors $p_{1}$ and $p_{2}$ : in other words, we obtain the same result for $M$ if we substitute $\left(p_{1}, p_{2}\right)$ with $\left(\alpha_{1} p_{1}, \alpha_{2} p_{2}\right), \alpha_{1}$ and $\alpha_{2}$ being arbitrary positive constants.

Therefore, $M$ is forced to have the following form:

$$
M\left(p_{1}, p_{2} ; t\right)=f_{M}\left(\frac{p_{1}}{\sqrt{p_{1}^{2}}} \cdot \frac{p_{2}}{\sqrt{p_{2}^{2}}} ; t\right)
$$


For analogous reasons, $E$ must be of the form:

$$
E\left(p_{1 E}, p_{2 E} ; t\right)=f_{E}\left(\frac{p_{1 E}}{\left|p_{1 E}\right|} \cdot \frac{p_{2 E}}{\left|p_{2 E}\right|} ; t\right)
$$

where $\left|B_{E}\right| \equiv \sqrt{\sum_{\mu=1}^{4} B_{E \mu}^{2}}$ is the Euclidean norm. (A short remark about the notation: we have denoted everywhere the scalar product by a ".", both in the Minkowski and the Euclidean world. Of course, when $A$ and $B$ are Minkowski four-vectors, then $A \cdot B=$ $A^{\mu} B_{\mu}=A^{0} B^{0}-\mathbf{A} \cdot \mathbf{B}$; while, if $A_{E}$ and $B_{E}$ are Euclidean four-vectors, then $A_{E} \cdot B_{E}=$ $A_{E \mu} B_{E \mu}=\mathbf{A}_{E} \cdot \mathbf{B}_{E}+A_{E 4} B_{E 4}$ ) Therefore, the relation (2.20) can be re-formulated as follows [observing that $\left.\left(p_{i E} / m\right) /\left|\left(p_{i E} / m\right)\right|=p_{i E} /\left|p_{i E}\right|\right]$

$$
f_{E}\left(v_{1 E} \cdot v_{2 E} ; t\right)=f_{M}\left(\bar{u}_{1} \cdot \bar{u}_{2} ; t\right)
$$

where $v_{1 E}$ and $v_{2 E}$ are the Euclidean four-versors corresponding to $p_{1 E}$ and $p_{2 E}\left(v_{1 E}^{2}=\right.$ $\left.v_{2 E}^{2}=1\right)$ :

$$
v_{1 E}=\frac{p_{1 E}}{\left|p_{1 E}\right|} \quad, \quad v_{2 E}=\frac{p_{2 E}}{\left|p_{2 E}\right|}
$$

while $\bar{u}_{1}$ and $\bar{u}_{2}$ are the Minkowski four-vectors defined as

$$
\bar{u}_{1}=\frac{\tilde{p}_{1}}{\sqrt{\tilde{p}_{1}^{2}}} \quad, \quad \bar{u}_{2}=\frac{\tilde{p}_{2}}{\sqrt{\tilde{p}_{2}^{2}}} .
$$

(It is clear that: $\bar{u}_{1}^{2}=\bar{u}_{2}^{2}=1$.) By virtue of the $O(4)$ symmetry of the Euclidean theory, we can choose a reference frame in which the spatial vectors $\mathbf{v}_{1 E}$ and $\mathbf{v}_{2 E}=-\mathbf{v}_{1 E}$ are along the $x_{1}$-direction. The two four-momenta $v_{1 E}$ and $v_{2 E}$ are, therefore,

$$
\begin{aligned}
& v_{1 E}=\left(\sin \phi, \mathbf{0}_{t}, \cos \phi\right) ; \\
& v_{2 E}=\left(-\sin \phi, \mathbf{0}_{t}, \cos \phi\right),
\end{aligned}
$$

where $\phi$ is the angle formed by each trajectory with the $x_{4}$-axis. The value of $\phi$ is between 0 and $\pi / 2$, so that the angle $\theta=2 \phi$ between the two Euclidean trajectories $W_{1 E}$ and $W_{2 E}$ lies in the range $[0, \pi]$ : it is always possible to make such a choice by virtue of the $O(4)$ symmetry of the Euclidean theory. In such a reference frame, we can write $v_{1 E} \cdot v_{2 E}=\cos \theta$. 
From Eq. (2.18) we have that $\tilde{p}_{i}^{2}=-\left|p_{i E}\right|^{2}<0$ and $\sqrt{\tilde{p}_{i}^{2}}=-i\left|p_{i E}\right|$. The sign of the squared root is fixed in the following way: in the system where $\mathbf{p}_{i}=\mathbf{0}$, we have that $\sqrt{p_{i}^{2}}=p_{i}^{0}$ (if we take $p_{i}^{0}>0$ ). This relation is continued so to have $\sqrt{\tilde{p}_{i}^{2}}=\tilde{p}_{i}^{0}$ in the system where $\tilde{\mathbf{p}}_{i}=\mathbf{0}$. But $\tilde{\mathbf{p}}_{i}=\mathbf{p}_{i E}=\mathbf{0}$, so that $\tilde{p}_{i}^{0}=-i p_{i E 4}=-i\left|p_{i E}\right|$ (if we take $\left.p_{i E 4}>0\right)$. Therefore, in this particular system $\sqrt{\tilde{p}^{2}}=\tilde{p}^{0}=-i p_{i E 4}=-i\left|p_{i E}\right|$. So we take $\sqrt{\tilde{p}^{2}}=-i\left|p_{i E}\right|$ in every system. This implies that:

$$
\bar{u}_{i}=\frac{\tilde{p}_{i}}{\sqrt{\tilde{p}_{i}^{2}}}=\left(v_{i E 4}, i \mathbf{v}_{i E}\right) .
$$

With the explicit form of $v_{1 E}$ and $v_{2 E}$ given by Eq. (2.26), we find that

$$
\begin{aligned}
& \bar{u}_{1}=\left(\cos \phi, i \sin \phi, \mathbf{0}_{t}\right), \\
& \bar{u}_{2}=\left(\cos \phi,-i \sin \phi, \mathbf{0}_{t}\right),
\end{aligned}
$$

and consequently $\bar{u}_{1}^{2}=\bar{u}_{2}^{2}=1$ and

$$
\bar{u}_{1} \cdot \bar{u}_{2}=\cos (2 \phi)=\cos \theta .
$$

A comparison with the expressions (2.5) for the Minkowski four-vectors $u_{1}$ and $u_{2}$ reveals that $\bar{u}_{1}$ and $\bar{u}_{2}$ are obtained from $u_{1}$ and $u_{2}$ after the following analytic continuation in the angular variables is made:

$$
\chi \rightarrow i \theta .
$$

(We remind that $\phi=\theta / 2$ and $\psi=\chi / 2$.) Therefore, by virtue of Eqs. (2.21) and (2.22), the relation $(2.23)$ can be formulated as follows:

$$
E(\theta ; t)=M(\chi \rightarrow i \theta ; t)
$$

Let us consider, now, the Wilson-line's renormalization constant $Z_{W}$ :

$$
Z_{W} \equiv \frac{1}{N_{c}}\left\langle\operatorname{Tr}\left[W_{1}(0)\right]\right\rangle
$$

We can expand $W_{1}$ in power series of $g$ and take the piece with $g^{n}$, whose contribution to $Z_{W}$ we call $Z_{W}^{(n)}$ :

$$
\begin{aligned}
Z_{W}^{(n)} & =\frac{(-i g)^{n}}{N_{c}} \operatorname{Tr}\left(T^{a_{1}} \ldots T^{a_{n}}\right) \int d \tau_{1} \frac{p_{1}^{\mu_{1}}}{m} \ldots \int d \tau_{n} \frac{p_{1}^{\mu_{n}}}{m} \times \\
& \times \theta\left(\tau_{n}-\tau_{n-1}\right) \ldots \theta\left(\tau_{2}-\tau_{1}\right)\left\langle A_{\mu_{1}}^{a_{1}}\left(\frac{p_{1}}{m} \tau_{1}\right) \ldots A_{\mu_{n}}^{a_{n}}\left(\frac{p_{1}}{m} \tau_{n}\right)\right\rangle .
\end{aligned}
$$


In the Euclidean theory we have, analogously:

$$
Z_{W E} \equiv \frac{1}{N_{c}}\left\langle\operatorname{Tr}\left[W_{1 E}(0)\right]\right\rangle_{E}
$$

and

$$
\begin{aligned}
& Z_{W E}^{(n)}=\frac{(-i g)^{n}}{N_{c}} \operatorname{Tr}\left(T^{a_{1}} \ldots T^{a_{n}}\right) \int d \tau_{1} p_{1 E}^{\mu_{1}} \ldots \int d \tau_{n} p_{1 E}^{\mu_{n}} \times \\
& \quad \times \theta\left(\tau_{n}-\tau_{n-1}\right) \ldots \theta\left(\tau_{2}-\tau_{1}\right)\left\langle A_{(E) \mu_{1}}^{a_{1}}\left(p_{1 E} \tau_{1}\right) \ldots A_{(E) \mu_{n}}^{a_{n}}\left(p_{1 E} \tau_{n}\right)\right\rangle_{E} .
\end{aligned}
$$

Using Eq. (2.13), we can derive the following relation:

$$
\begin{aligned}
\frac{\tilde{p}_{1}^{\mu_{1}}}{m} & \ldots \frac{\tilde{p}_{1}^{\mu_{n}}}{m}\left\langle A_{\mu_{1}}^{a_{1}}\left(\frac{\tilde{p}_{1}}{m} \tau_{1}\right) \ldots A_{\mu_{n}}^{a_{n}}\left(\frac{\tilde{p}_{1}}{m} \tau_{n}\right)\right\rangle= \\
\quad & =\frac{p_{1 E}^{\mu_{1}}}{m} \ldots \frac{p_{1 E}^{\mu_{n}}}{m}\left\langle A_{(E) \mu_{1}}^{a_{1}}\left(\frac{p_{1 E}}{m} \tau_{1}\right) \ldots A_{(E) \mu_{n}}^{a_{n}}\left(\frac{p_{1 E}}{m} \tau_{n}\right)\right\rangle_{E},
\end{aligned}
$$

where, as usual, $p_{1 E}=\left(\mathbf{p}_{1 E}, p_{1 E 4}\right)$ and $\tilde{p}_{1}=\left(\tilde{p}_{1}^{0}, \tilde{\mathbf{p}}_{1}\right)=\left(-i p_{1 E 4}, \mathbf{p}_{1 E}\right)$. If we define

$$
\begin{array}{ccc}
Z_{W} \equiv h_{M}\left(p_{1}\right) & \quad Z_{W}^{(n)}=h_{M}^{(n)}\left(p_{1}\right), \\
Z_{W E} \equiv h_{E}\left(p_{1 E}\right) & , & Z_{W E}^{(n)}=h_{E}^{(n)}\left(p_{1 E}\right)
\end{array}
$$

from Eq. (2.36) we obtain

$$
h_{E}^{(n)}\left(\frac{p_{1 E}}{m}\right)=h_{M}^{(n)}\left(\tilde{p}_{1}\right) .
$$

This relation is valid for every integer number $n$ and so we also have, more generally:

$$
h_{E}\left(\frac{p_{1 E}}{m}\right)=h_{M}\left(\tilde{p}_{1}\right)
$$

From the definitions (2.32) and (2.2), $h_{M}\left(p_{1}\right)$, considered as a function of a general fourvector $p_{1}$, is a scalar function constructed with the only four-vector $p_{1}$. In addition, $h_{M}\left(p_{1}\right)$ does not depend on the (positive) normalization of $p_{1}$ : in other words, $h_{M}\left(\alpha p_{1}\right)=h_{M}\left(p_{1}\right)$ for every positive $\alpha$. Therefore, $h_{M}\left(p_{1}\right)$ is forced to have the form

$$
h_{M}\left(p_{1}\right)=H_{M}\left(u_{1}^{2}\right)=H_{M}(1)
$$

where $u_{1}=p_{1} / \sqrt{p_{1}^{2}}\left(u_{1}^{2}=1\right)$. In a perfectly analogous way, for the Euclidean case we have that:

$$
h_{E}\left(p_{1 E}\right)=H_{E}\left(v_{1 E}^{2}\right)=H_{E}(1)
$$


where $v_{1 E}=p_{1 E} /\left|p_{1 E}\right|\left(v_{1 E}^{2}=1\right)$. Therefore, the first member of Eq. (2.39) is just equal to $h_{E}\left(p_{1 E} / m\right)=H_{E}\left(v_{1 E}^{2}\right)=H_{E}(1)$ [observing that $\left.\left(p_{1 E} / m\right) /\left|\left(p_{1 E} / m\right)\right|=p_{1 E} /\left|p_{1 E}\right|\right]$, and the second member is given by $h_{M}\left(\tilde{p}_{1}\right)=H_{M}\left(\bar{u}_{1}^{2}\right)=H_{M}(1)$, where $\bar{u}_{1}=\tilde{p}_{1} / \sqrt{\tilde{p}_{1}^{2}}\left(\bar{u}_{1}^{2}=1\right)$. Then Eq. (2.39) implies that

$$
H_{E}(1)=H_{M}(1) \text {. }
$$

That is, from Eqs. (2.37), (2.40) and (2.41):

$$
Z_{W E}=Z_{W}
$$

Combining this identity with Eq. (2.31), we find the following relation between the amplitudes $g_{M}(\chi ; t)=M(\chi ; t) / Z_{W}^{2}$ and $g_{E}(\theta ; t)=E(\theta ; t) / Z_{W E}^{2}$ :

$$
\begin{aligned}
g_{M}(\chi ; t) \underset{\chi \rightarrow i \theta}{\longrightarrow} g_{M}(i \theta ; t) & =g_{E}(\theta ; t) ; \\
\text { or : } g_{E}(\theta ; t) \underset{\theta \rightarrow-i \chi}{\longrightarrow} g_{E}(-i \chi ; t) & =g_{M}(\chi ; t) .
\end{aligned}
$$

We have derived the relation (2.44) of analytic continuation for a non-Abelian gauge theory with gauge group $S U\left(N_{c}\right)$. It is clear, from the derivation given above, that the same result is valid also for an Abelian gauge theory (QED). We have thus completely generalized the results of Ref. [4], where the same relation (2.44) had been proven for an Abelian gauge theory (QED) in the so-called quenched approximation and for a nonAbelian gauge theory (QCD) up to the fourth order in the renormalized coupling constant in perturbation theory. The approach adopted in Ref. [- consisted in explicitly evaluating the amplitudes $g_{M}(\chi ; t)$ and $g_{E}(\theta ; t)$, in the Minkowski and the Euclidean world, in some given approximation (such as the quenched approximation) or up to some order in perturbation theory and in finally comparing the two expressions so obtained. Instead, in this paper we have given a general proof of Eq. (2.44), which essentially exploits the relation (2.13) between the gluonic Green functions in the two theories.

Therefore, it is possible to reconstruct the high-energy scattering amplitude by evaluating a correlation of two infinite Wilson lines forming a certain angle $\theta$ in Euclidean four-space, then by continuing this quantity in the angular variable, $\theta \rightarrow-i \chi$, where $\chi$ is the hyperbolic angle between the two Wilson lines in Minkowski space-time, and finally by performing the limit $\chi \rightarrow \infty$ (i.e., $\beta \rightarrow 1$ ). In fact, the high-energy scattering amplitude is given by

$$
\begin{aligned}
& M_{f i}=\left\langle\psi_{i \alpha}\left(p_{1}^{\prime}\right) \psi_{k \gamma}\left(p_{2}^{\prime}\right)|M| \psi_{j \beta}\left(p_{1}\right) \psi_{l \delta}\left(p_{2}\right)\right\rangle \\
& \underset{s \rightarrow \infty}{\sim}-i \cdot 2 s \cdot \delta_{\alpha \beta} \delta_{\gamma \delta} \cdot g_{M}(\chi \rightarrow \infty ; t) .
\end{aligned}
$$


The quantity $g_{M}(\chi ; t)$, defined by Eq. (2.1) in the Minkowski world, is linked to the corresponding quantity $g_{E}(\theta ; t)$, defined by Eq. (2.7) in the Euclidean world, by the analytic continuation (2.44) in the angular variables. The important thing to note here is that the quantity $g_{E}(\theta ; t)$, defined in the Euclidean world, may be computed non perturbatively by well-known and well-established techniques, for example by means of the formulation of the theory on the lattice. In all cases, once one has obtained the quantity $g_{E}(\theta ; t)$, one still has to perform an analytic continuation in the angular variable $\theta \rightarrow-i \chi$, and finally one has to extrapolate to the limit $\chi \rightarrow \infty$ (i.e., $\beta \rightarrow 1$ ). For deriving the dependence on $s$ one exploits the fact that both $\beta$ and $\psi$ (or equivalently $\chi$ ) are dependent on $s$. In fact, from $E=m / \sqrt{1-\beta^{2}}$ and from $s=4 E^{2}$, one immediately finds that

$$
\beta=\sqrt{1-\frac{4 m^{2}}{s}} .
$$

By inverting this equation and using the relation $\beta=\tanh \psi$, we derive:

$$
s=4 m^{2} \cosh ^{2} \psi=2 m^{2}(\cosh \chi+1) .
$$

Therefore, in the high-energy limit $s \rightarrow \infty$ (or $\beta \rightarrow 1$ ), the hyperbolic angle $\chi=2 \psi$ is essentially equal to the logarithm of $s$ (for a finite non-zero quark mass $m$ ):

$$
\chi=2 \psi \underset{s \rightarrow \infty}{\sim} \ln s .
$$

As an example, we have shown in Ref. [4] how, using this approach, one can re-derive the well-known Regge Pole Model [11]. Of course, the most interesting results are expected from an exact non perturbative approach, for example by directly computing $g_{E}(\theta ; t)$ on the lattice: a considerable progress could be achieved along this direction in the near future. 


\section{References}

[1] O. Nachtmann, Ann. Phys. 209, 436 (1991).

[2] E. Meggiolaro, Phys. Rev. D 53, 3835 (1996).

[3] H. Verlinde and E. Verlinde, Princeton University, report No. PUPT-1319 (revised 1993); hep-th/9302104.

[4] E. Meggiolaro, Pisa preprint, IFUP-TH 10/96 (1996); hep-th/9602104; Zeitschrift für Physik C (1997), currently in press.

[5] H. Cheng and T.T. Wu, Expanding Protons: Scattering at High Energies (MIT Press, Cambridge, Massachussets, 1987).

[6] L.N. Lipatov, in Review in Perturbative QCD, edited by A.H. Mueller (World Scientific, Singapore, 1989), and references therein.

[7] I.Ya. Aref'eva, Phys. Lett. 93B, 347 (1980).

[8] G.P. Korchemsky, Phys. Lett. B 325, 459 (1994); I.A. Korchemskaya and G.P. Korchemsky, Nucl. Phys. B437, 127 (1995).

[9] I.Ya. Aref'eva, Phys. Lett. B 325, 171 (1994); 328, 411 (1994).

[10] H.G. Dosch, E. Ferreira and A. Krämer, Phys. Rev. D 50, 1992 (1994).

[11] T. Regge, Nuovo Cimento 14, 951 (1959); 18, 947 (1960). 